\title{
COMPARAÇÃO DE VEDAÇÕES LATERAIS EM PRENSAS DE ROLOS VIA SIMULAÇÃO PELO MÉTODO DOS ELEMENTOS DISCRETOS*
}

\author{
Gilvandro Bueno ${ }^{1}$ \\ Gabriel Kamilo Pantoja Barrios ${ }^{2}$ \\ Frederico Correa Mayerhofer ${ }^{3}$ \\ Rodrigo Magalhães de Carvalho ${ }^{4}$ \\ Luís Marcelo Marques Tavares ${ }^{4}$
}

\begin{abstract}
Resumo
Atualmente, diferentes tipos de vedações laterais de rolos, usados para limitar o efeito de borda em prensas de rolos "High Pressure Grinding Rolls (HPGR)" se encontram disponíveis. O presente trabalho comparou dois métodos usados na vedação lateral: as placas laterais (cheek plates) e as flanges, essas últimas colocadas em um dos dois rolos, a partir de simulações usando o Método dos Elementos Discretos "Discrete Element Method (DEM)", com acoplamento dinâmico multicorpos (Multibody dynamics ou MBD). Como resultado das simulações foram obtidos os perfis de força compressiva ao longo do rolo, assim como a proporção do fluxo mássico de alimentação que é projetado fora da zona de compressão durante a prensagem. As simulações mostraram que ...do HPGR usando o DEM + MBD se apresentam como uma ferramenta muito útil para visualizar o melhor modelo de vedação de modo a minimizar o efeito de borda durante o processo de prensagem.

Palavras-chave: DEM; Efeito de borda; HPGR.
\end{abstract}

\section{COMPARISON OF LATERAL SEALS IN ROLLER PRESSES VIA SIMULATION BY THE DISCRETE ELEMENTS METHOD}

\begin{abstract}
Presently, different types of lateral seals are used to limit the edge effect in High Pressure Grinding Rolls (HPGR) or roller presses. The present work compared two methods used in this task: the cheek plates and the flanges, the latter placed on one of the two rollers, from simulations using the Discrete Element Method (DEM), with multibody dynamics (MBD). As result of the simulations, the profiles of compressive force along the roll were obtained, as well as the proportion of the mass feed stream that is projected outside the compression zone during pressing. The simulations of HPGR using DEM + MBD are presented as a very useful tool to visualize the best sealing model to minimize the edge effect during the pressing process.
\end{abstract}

Keywords: DEM; Edge effect; HPGR.

1 Mestrando no Programa de Engenharia Metalúrgica e Materiais - COPPE - UFRJ - Engenheiro de Processo na Vale - ES

Graduando em Engenharia Metalúrgica e Materiais - Escola Politécnica - UFRJ.

Gerente de Engenharia de Processo na Vale - ES

Professor Adjunto do Programa de Engenharia Metalúrgica e Materiais - COPPE - UFRJ.

Professor Titular do Programa de Engenharia Metalúrgica e Materiais - COPPE - UFRJ. 


\section{INTRODUÇÃO}

A pelotização surgiu como uma alternativa para o aproveitamento de minérios de ferro fino (pellet feed) que são finos demais para serem alimentados nos fornos de redução direta ou altos fornos. Dentro do processo de pelotização de minério de ferro, a prensagem em prensa de rolos (High Pressure Grinding Rolls - HPGR) é considerada uma etapa muito importante, sendo responsável pelo aumento da área superficial do pellet feed antes do processo de formação da pelota verde, e queima. A Figura 1 apresenta o fluxograma simplificado de usina de pelotização, enquanto a Figura 2 mostra um HPGR.
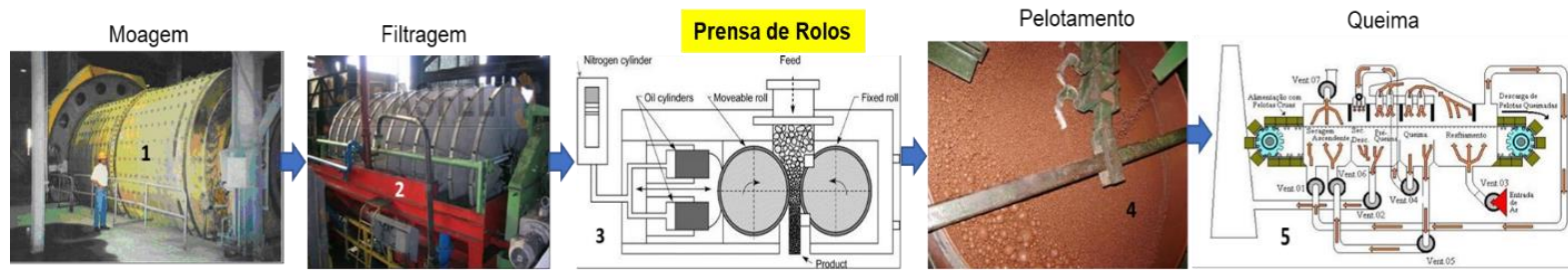

Figura 1. Fluxograma esquemático de usina de pelotização

A tecnologia de fragmentação por HPGR tem ganhado espaço na indústria de transformação mineral em todo mundo, face à competitividade dos custos operacionais envolvidos neste equipamento quando comparado as outras alternativas (TAVARES, 2005; VAN DER MEER, 2010). Mesmo com a experiência desenvolvida ao longo do tempo, não se chegou ao entendimento pleno do desempenho deste equipamento, sendo que algumas perguntas ainda precisam ser respondidas. Assim, a simulação pode ser uma ferramenta poderosa para prever e comparar cenários futuros com relativo baixo custo associado.

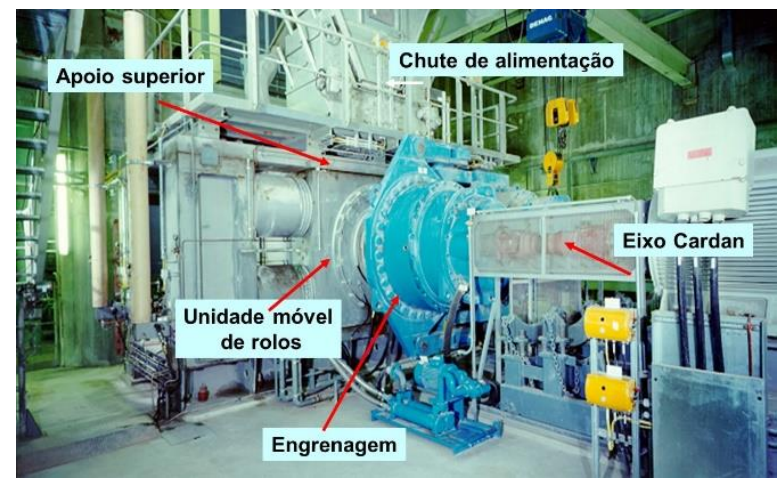

Figura 2. Prensa de rolos (HPGR) Fonte: Brochure Polysius

Avanços importantes na modelagem da fragmentação têm sido desenvolvidos, sendo que os mais recentes, denominados de modelos mecanicistas, utilizam os Métodos de Elementos Discretos (DEM), como parte importante de sua concepção (MISHRA e RAJAMANI,1992; CARVALHO, 2009).

O modelo UFRJ, inicialmente concebido como um modelo generalizado da cominuição (MGC), combina informações do ambiente de moagem (ou britagem) às equações de balanço de massa derivadas do modelo do balanço populacional e tem sido aplicado na descrição de diferentes equipamentos:

- Moinhos de bolas (TAVARES; CARVALHO, 2009);

- Moinhos semiautógenos (CARVALHO, 2014);

- Britadores verticais de impacto (DA CUNHA; CARVALHO; TAVARES, 2013);

- Degradação de partículas durante o manuseio (TAVARES; CARVALHO, 2011). 
Entretanto, a descrição usando ferramentas DEM de equipamentos de cominuição como britadores cônicos e prensa de rolos, deve considerar a interação dinâmica das geometrias do equipamento que estão em contato com as partículas submetidas a esforços (BARRIOS, 2015).

Especificamente, no caso da prensa de rolos, a modelagem da dinâmica do rolo móvel é importante para a correta descrição do desempenho do equipamento usando a simulação DEM. BARRIOS (2015) desenvolveu simulações DEM do HPGR usando um acoplamento de duas vias entre um código que simula a dinâmica de multicorpos do rolo móvel do HPGR com o software EDEM ${ }^{\circledR}$. Variáveis do desempenho do HPGR como capacidade, potencia, abertura de trabalho e perfil de pressão ao longo dos rolos, são alguns dos resultados obtidos nas simulações DEM. No que diz respeito ao funcionamento do HPGR, diversos autores descrevem que existe um perfil característico de pressão ao longo do rolo (Figura 3). Este perfil gera o que se define como efeito de borda. O gradiente de pressão é originado da descarga de material nas laterais do rolo (WATSON e BROOKS, 1994). O chamado "efeito de borda" é explicado pelo gradiente de pressão ao longo do comprimento do rolo, e à ausência de confinamento lateral do leito de partículas nas bordas dos rolos (DANIEL E MORREL, 2004).

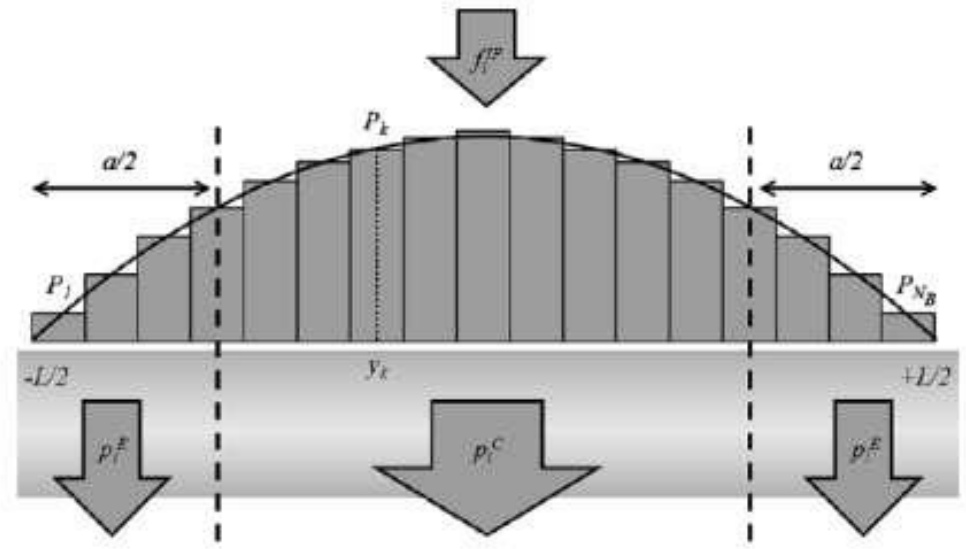

Figura 3. Perfil de pressão ao longo do rolo (TORRES e CASALI, 2009)

A forma tradicional de vedação lateral, utilizada pela maioria dos fabricantes de HPGR consiste do uso dos cheek plates, que são placas de encosto fixas posicionadas nas laterais dos rolos, responsáveis por confinar o minério na zona de prensagem entre os rolos, conforme visualizado na Figura 4.
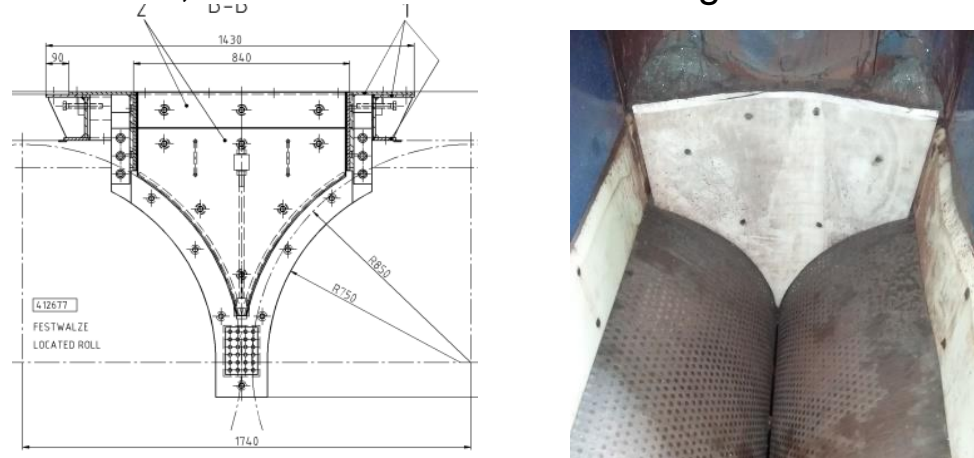

Figura 4. Cheek Plates - Fonte: Polysius - Vale

Uma outra forma de vedação consiste na inserção de um flange na forma de aro no próprio rolo em um dos rolos de modo que o outro rolo fique confinado dentro do rolo 
que possui o flange (Figura 5). O problema de perda de pressão nas bordas pode ser atenuado mediante a inserção de aros laterais, confinando o leito e prevenindo fluxo de material do leito no sentido longitudinal (ALVES, 2012).

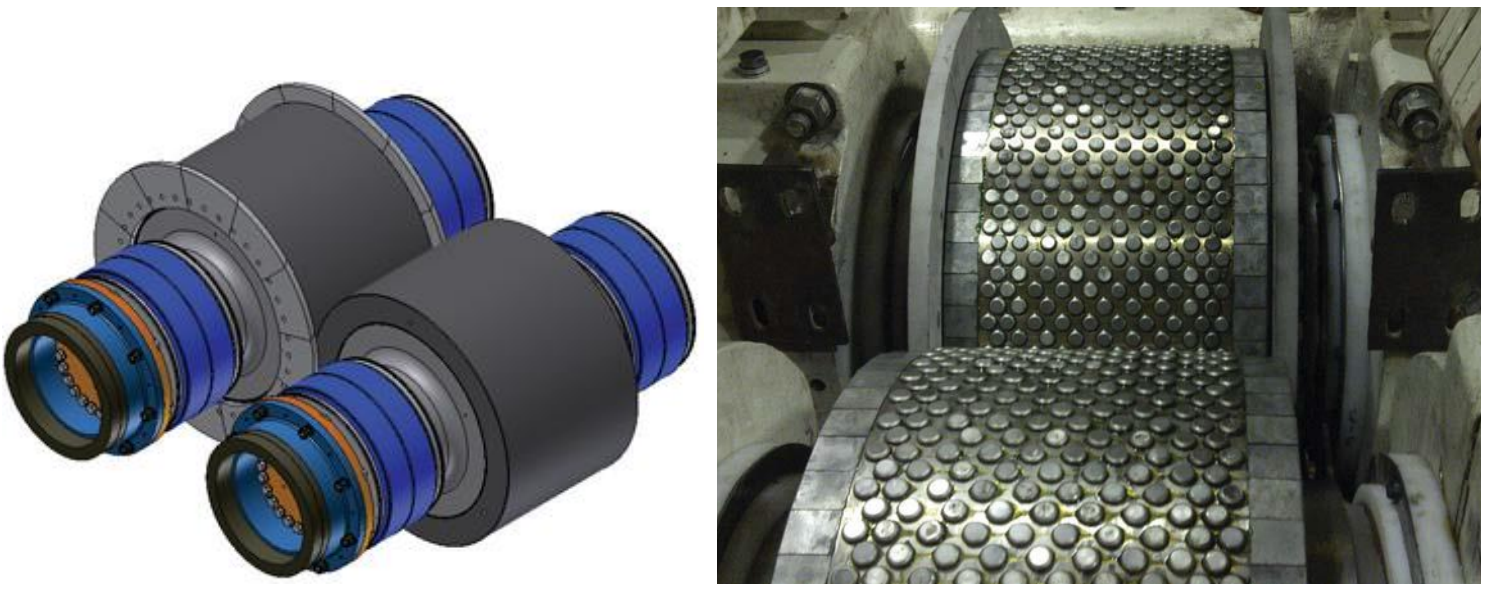

Figura 5. Rolos com flange de vedação Fonte: Brochure Metso

O presente trabalho teve como objetivo a avaliação das duas formas de vedação tendo como base a análise dos esforços sobre os rolos a partir de simulações realizadas por meio do Método dos Elementos Discretos (DEM).

\section{MATERIAIS E MÉTODOS}

Para a realização das simulações foi utilizado o software EDEM ${ }^{\circledR} 2.7$ (DEM Solutions, 2016).

\subsection{Características da simulação DEM do HPGR}

No presente trabalho foi simulado um HPGR em escala de laboratório. O modelo 3D foi construído com base em informações do manual do fabricante. O HPGR foi simulado em escala real com diâmetro dos rolos de $250 \mathrm{~mm}$ e $100 \mathrm{~mm}$ de comprimento. Optou-se por simular a prensa de laboratório e não a industrial de forma a reduzir o número de partículas no sistema, e, portanto, reduzir o esforço computacional necessário para realização das simulações.

A alimentação dos rolos na aplicação em questão é o pellet feed, o qual é formado por partículas menores que 0,150 milímetros, sendo que os cheek plates estão normalmente a uma distância entre 3 e 5 milímetros dos rolos. No caso dos rolos com flanges, existe uma folga de aproximadamente 5 milímetros entre o rolo interno e o flange.

As simulações no DEM da operação do HPGR realizadas no presente trabalho consideram a dinâmica do rolo móvel simulado por meio do acoplamento dinâmico multicorpos desenvolvido no Laboratório de Tecnologia Mineral (BARRIOS e TAVARES, 2016).

As simulações de diferentes tipos de vedações laterais foram realizadas de forma exploratória. Assim, de forma a diminuir o número de partículas, o tempo de simulação e o esforço computacional foram consideradas partículas esféricas de tamanho único (2,5 mm de diâmetro), obtendo simulações com menos de 50.000 partículas, possíveis de realizar em 48 h com um computador de 4 núcleos.

Foram selecionadas duas distancias em relação ao rolo, dos cheek plates e flanges para realização das simulações: a primeira com 2 milímetros, de modo a conter 
completamente as partículas na região de prensagem; e outra com 7,5 milímetros, proporcionando um certo grau de liberdade que representa 3 vezes o tamanho das partículas entre o rolo e o mecanismo de contenção. Nas Figuras 6 e 7 são apresentadas as geometrias em 3D dos rolos e vedações laterais (Cheek plates e Flanges) do HPGR modeladas no software SketchUp ${ }^{\circledR}$, as quais forma usadas nas simulações em DEM.

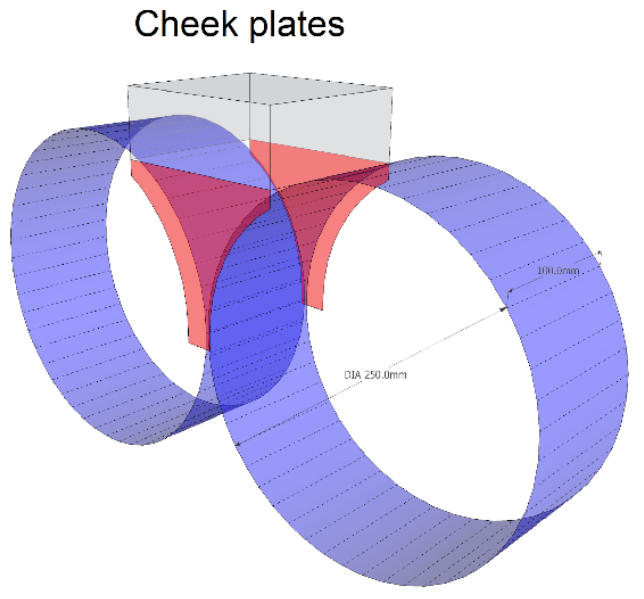

Figura 6. HPGR com Cheek Plates
Flanges

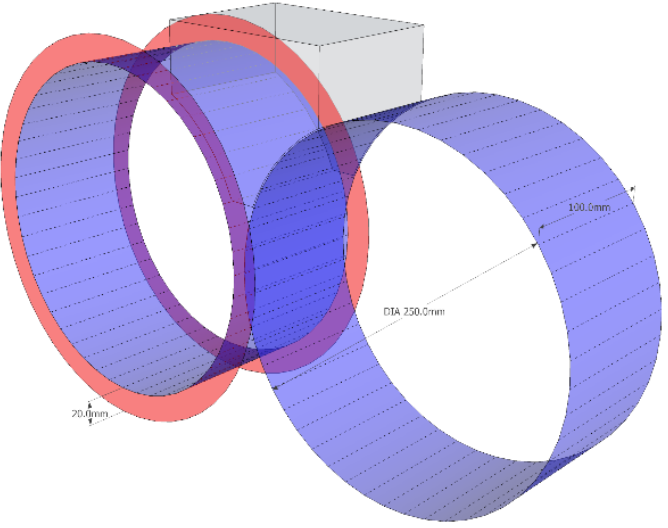

Figura 7. HPGR com Flanges

\subsection{Parâmetros utilizados nas simulações em DEM}

Para que a simulação em DEM tenha sucesso é necessário que os parâmetros sejam escolhidos de forma criteriosa visando a reprodução do comportamento físico (CARVALHO e BOECHAT, 2016). O software EDEM ${ }^{\circledR}$ oferece diferentes modelos de contatos que descrevem o comportamento das partículas e/ou geometrias quando entram em contato. O modelo de contato utilizado foi o modelo de Hertz-Mindlin (no slip) que é o modelo padrão para descrever os contatos partícula partículas de minério e geometria em aço. $\mathrm{O} \mathrm{EDEM}^{\circledR}$ permite acoplar modelos de contato customizados. Barrios (2015) desenvolveu um acoplamento ao modelo para descrever a dinâmica do rolo móvel. Este modelo considera a velocidade de rotação dos rolos fixo e móvel constantes, enquanto a dinâmica translacional do rolo móvel é descrita por meio do modelo de um sistema harmônico de massa e mola hidráulica.

Os parâmetros considerados na simulação são listados nas Tabelas 1 a 3 , tendo sido obtidos por Barrios (2015). Além dos itens descritos acima, ainda foram considerados a velocidade dos rolos em 4,8 rad./s $(0,66 \mathrm{~m} / \mathrm{s})$, uma fábrica gerando 57.558 partículas equivalente a 3,3 segundos de simulação com integração de $10^{-7}$ segundos e uma taxa de alimentação de $1,1 \mathrm{t} / \mathrm{h}$.

Tabela 1. Propriedades Individuais

\begin{tabular}{|c|c|c|}
\cline { 2 - 3 } \multicolumn{1}{c|}{ Parâmetros } & Aço & Minério \\
\hline Coeficiente de Poisson & 0,30 & 0,25 \\
\hline Módulo de Cisalhamento $(\mathrm{Pa})$ & $8,00 \mathrm{E}+10$ & $2,00 \mathrm{E}+09$ \\
\hline Densidade $\left(\mathrm{kg} / \mathrm{m}^{3}\right)$ & 7800 & 3948 \\
\hline
\end{tabular}


Tabela 2. Parâmetros de Contato

\begin{tabular}{|c|c|c|c|}
\cline { 2 - 4 } \multicolumn{1}{c|}{ Parâmetros } & Aço-Aço & Aço-Minerio & Minerio-Minerio \\
\hline Restitução & 0,7 & 0,38 & 0,48 \\
\hline Atrito & 0,2 & 0,5 & 0,49 \\
\hline Rolamento & 0,01 & 0,25 & 0,21 \\
\hline
\end{tabular}

Tabela 3. Parâmetros de Acoplamento

\begin{tabular}{|c|c|}
\cline { 2 - 2 } \multicolumn{1}{c|}{ Parâmetros } & Valores \\
\hline Pressão de Nitrogênio (bar) & 70 \\
\hline Pressão de Óleo (bar) & 100 \\
\hline Volume de Nitrogênio $\left(\mathrm{cm}^{3}\right)$ & 2000 \\
\hline Número de Pistões & 2 \\
\hline Área dos Pistões $\left(\mathrm{cm}^{2}\right)$ & 62 \\
\hline
\end{tabular}

\subsection{Metodologia Experimental}

Bueno e Vianna (2016) realizaram um experimento através de amostragem em usina industrial na Vale com o objetivo de verificar, na prática, o efeito de borda relacionado com 0 ganho de superfície específica, determinada pelo permeabilímetro de Blaine. $O$ trabalho experimental foi realizado colhendo-se amostras na alimentação da prensa, em cinco pontos abaixo do rolo, nas bordas e no produto final da prensa, sendo este último composto por todo material alimentado ao HPGR. A Figura 8 ilustra o fluxo de material no HPGR, incluindo material que sai pelas bordas, enquanto a Figura 9 mostra os pontos de amostragens experimentais. A prensa amostrada utiliza vedação lateral com Cheek Plates, tendo diâmetro de $1700 \mathrm{~mm}$ e comprimento de $1400 \mathrm{~mm}$, sendo acionada com motor de $1.430 \mathrm{~kW}$.

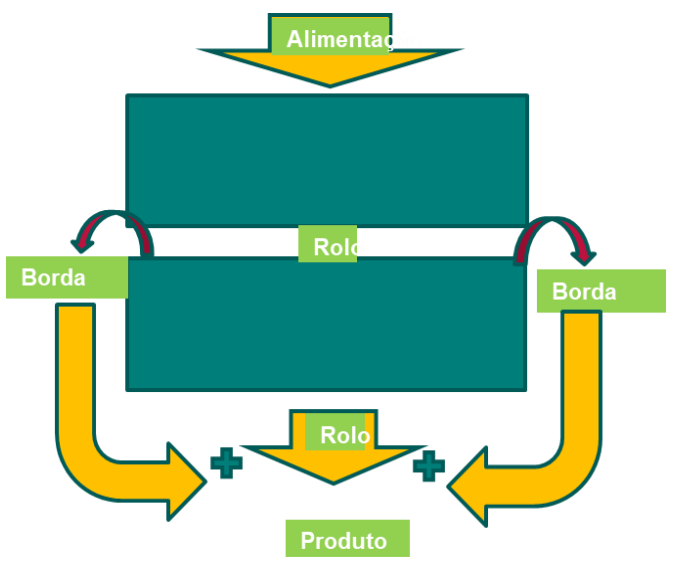

Figura 8. Fluxo de material pelo HPGR

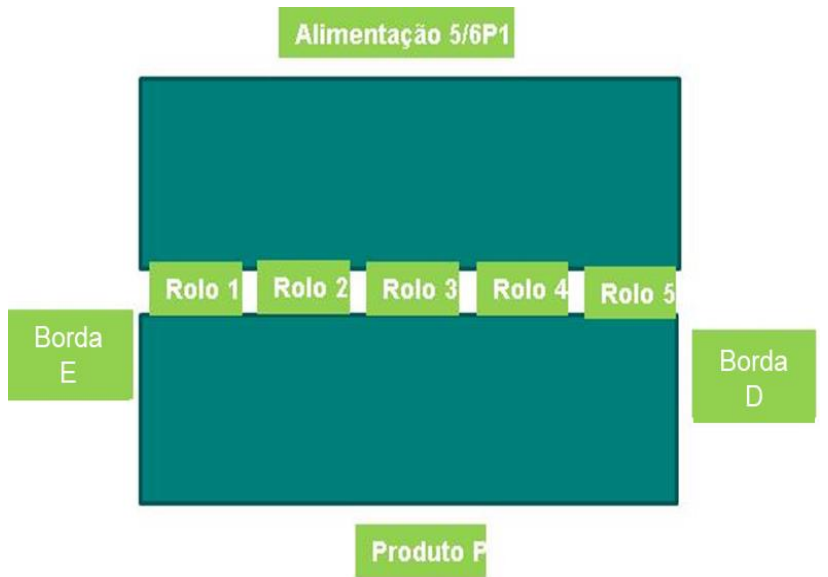

Figura 9. Pontos de Amostragens 
As condições de operação durante as amostragens são listadas na Tabela 4.

Tabela 4. Parâmetros durante amostragem

\begin{tabular}{|l|c|}
\hline \multicolumn{1}{|c|}{ Parâmetros } & Valores \\
\hline Taxa de Alimentação $(\mathrm{t} / \mathrm{h})$ & 632 \\
\hline GAP de trabalho $(\mathrm{mm})$ & 12 \\
\hline Velocidade periférica $(\mathrm{m} / \mathrm{s})$ & 1,4 \\
\hline Força Específica $\left(\mathrm{N} / \mathrm{mm}^{2}\right)$ & 2,2 \\
\hline
\end{tabular}

\section{RESULTADOS E DISCUSSÃO}

Após realizadas as simulações, o software EDEM ${ }^{\circledR}$ permitiu extrair as informações através de vídeos, imagens, gráficos ou mesmos dados que podem ser convertidos em planilhas. A Figura 10 ilustra imagens da simulação.

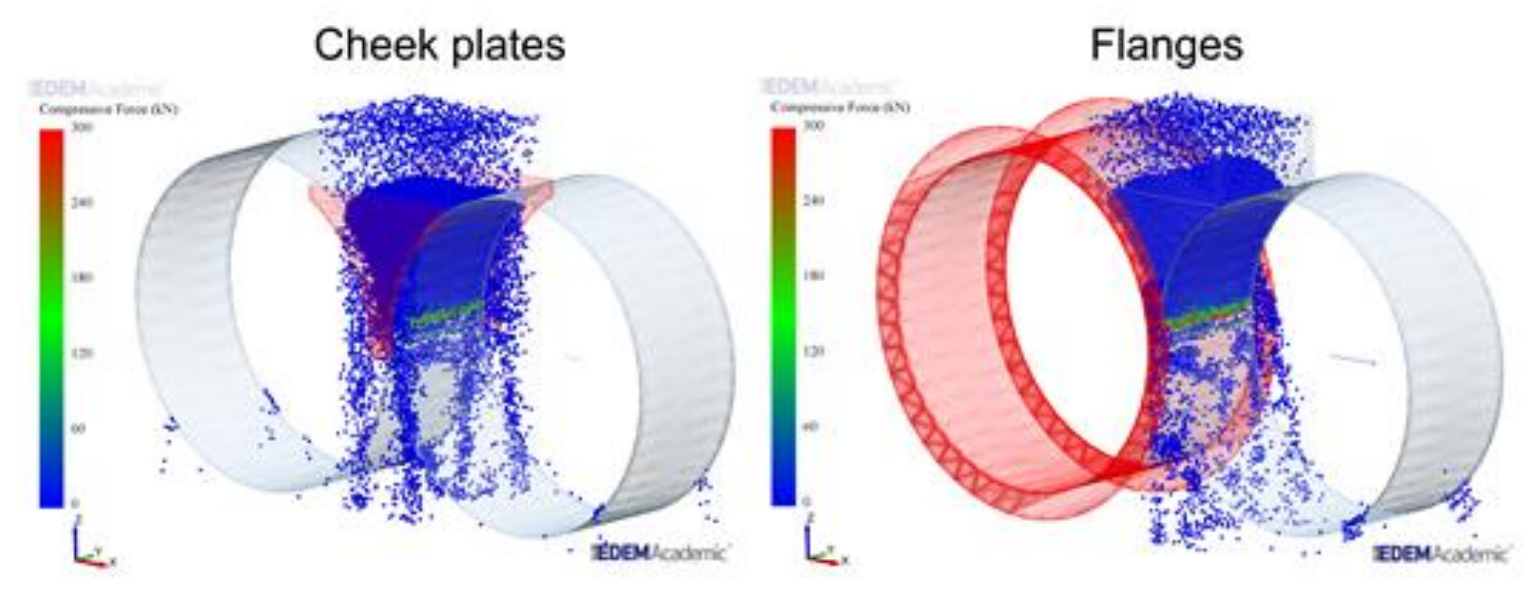

Figura 10. Simulações DEM do HPGR com diferentes vedações laterais com distância de 7,5 mm entre os rolos: Cheek Plates (esquerda) e Flanges (direita).

Dentre as diversas informações disponíveis no software, neste trabalho avaliou-se a força compressiva para verificação do perfil e o efeito de borda. Na Figura 11 se observa o comportamento da força compressiva no leito de partículas ao longo do rolo para as simulações com distância de 7,5 mm entre as vedações e os rolos. 

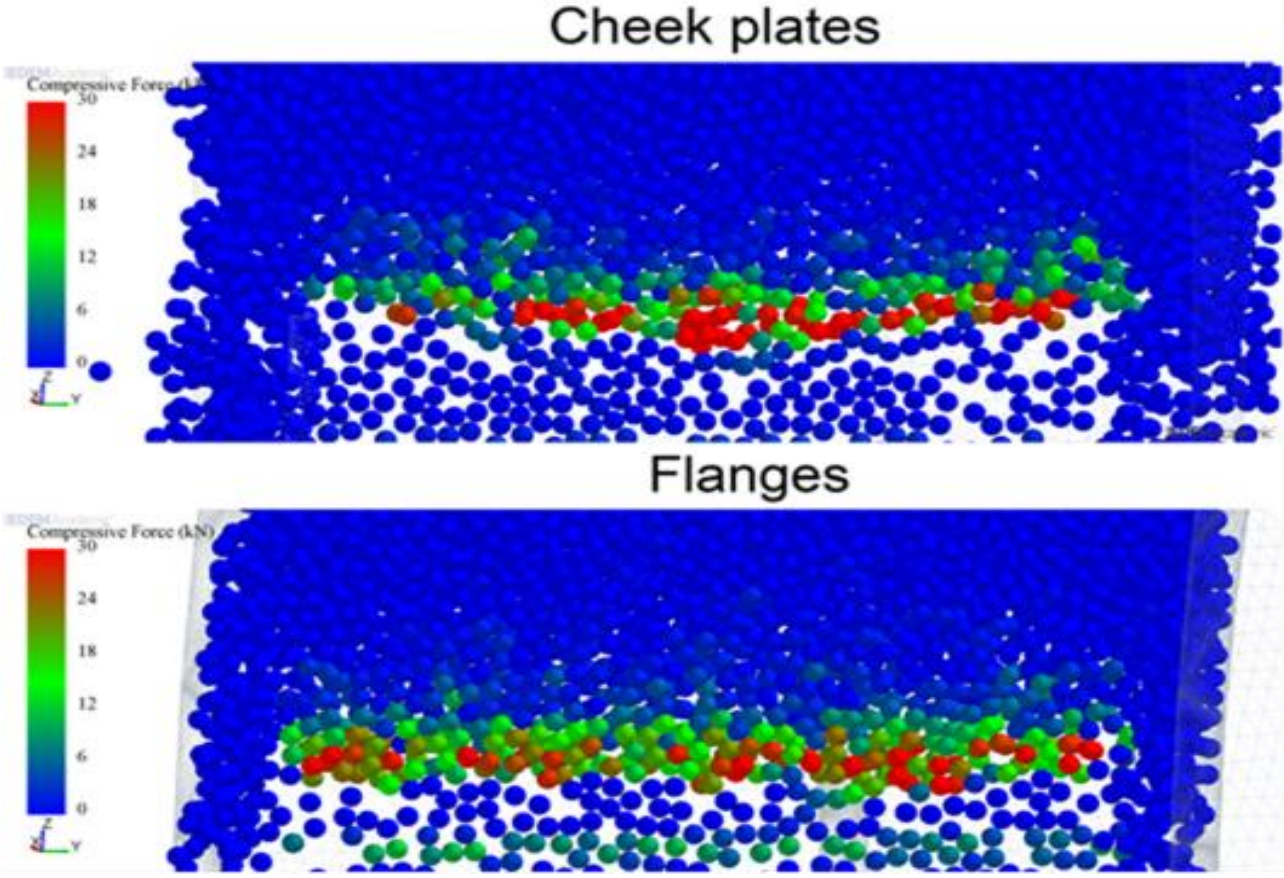

Figura 11. Visualização da força compressiva no leito de partículas longitudinalmente no rolo para os dois tipos de vedações laterais com distância de $7,5 \mathrm{~mm}$.

A partir dos dados extraídos da simulação foi possível analisar a distribuição dos esforços ao longo dos rolos e, portanto, verificar a eficiência de cada um dos sistemas de vedação comparados. A Figura 12 mostra os perfis de força do cheek plates e flange a uma distância de $7,5 \mathrm{~mm}$ do rolo, equivalente ao tamanho de três partículas, esta distância foi suficiente para dar liberdade às partículas e verificar o efeito de borda que ocorre no processo de prensagem na prensa de rolos.

É possível observar que o sistema com flange é capaz de garantir um perfil de forças mais elevado, quando comparado aos cheek plates. No entanto em ambos os casos o perfil característico do efeito de borda é observado.

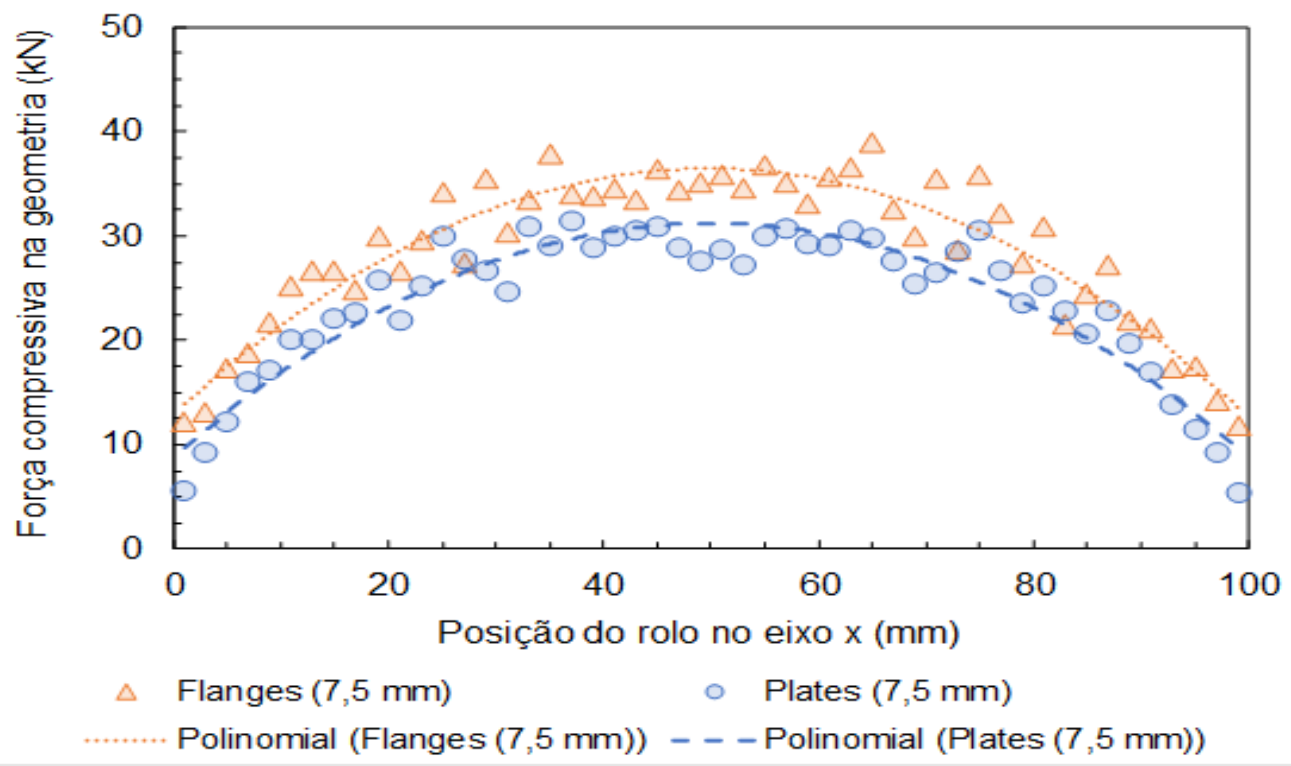

Figura 12. Perfis de força compressiva longitudinalmente no rolo para os dois tipos de vedações laterais com distância de $7,5 \mathrm{~mm}$ 
Foram realizadas também simulações com os flanges e cheek plates a $2 \mathrm{~mm}$ de distância em relação ao rolo, ou seja, sem liberdade para escape da partícula que foi definida com o diâmetro de $2,5 \mathrm{~mm}$. Nestas condições não se verifica o efeito de borda. Isso indica que, para um melhor desempenho da prensagem ao longo do rolo, esta vedação deve ser a mais perfeita possível. Na Figura 13 observa-se um perfil de força linear ao longo do rolo com cheek plates, adicionalmente, os resultados mostram que o flange gera um perfil com forças de maior magnitude nas bordas. Este fato pode ser explicado por esforços tangenciais que os flanges aplicam às partículas, porém o estudo deste efeito não foi contemplado neste trabalho.

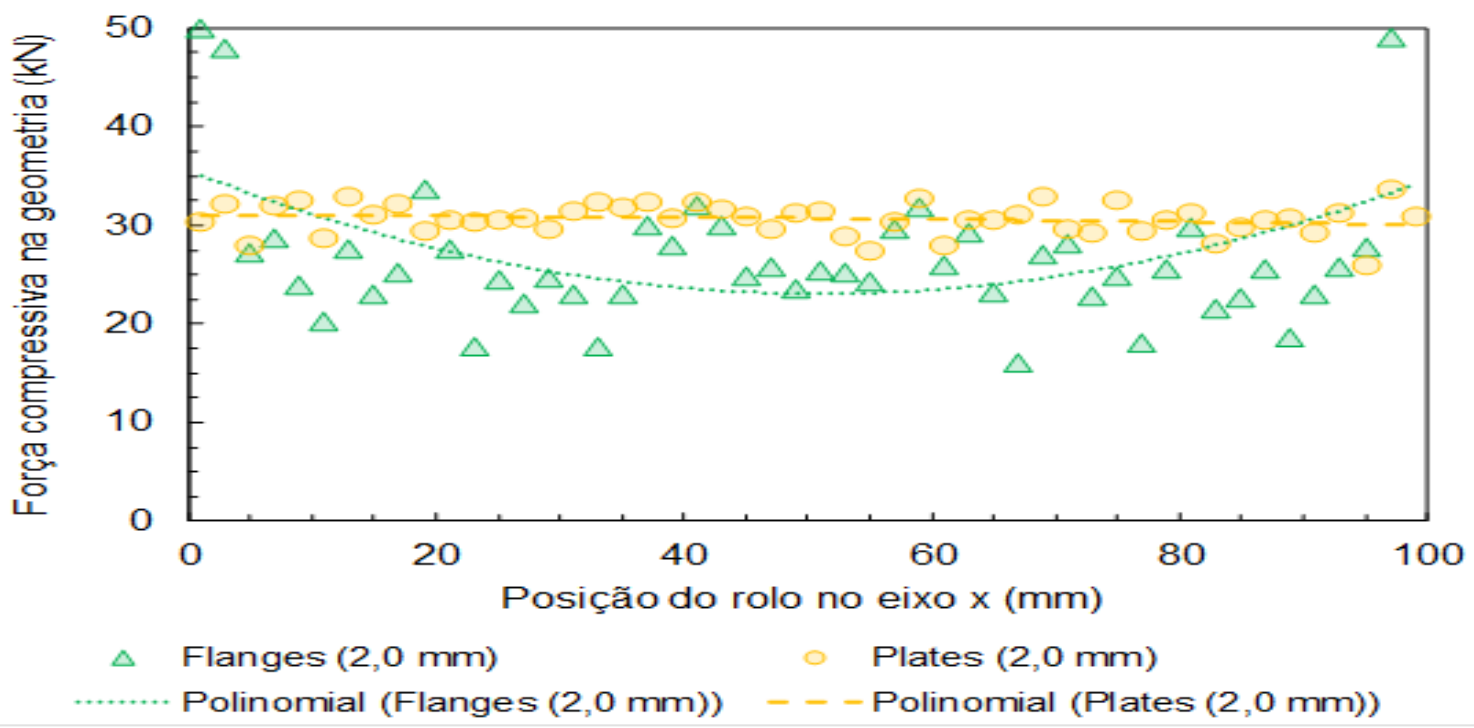

Figura 13. Perfis de força compressiva longitudinalmente no rolo para os dois tipos de vedações laterais com distância de 2,0 mm.

As simulações DEM do HPGR com diferentes vedações laterais também mostram informações importante sobre a velocidade das partículas que atravessam a zona de compressão e sobre a distribuição do fluxo mássico ao longo dos rolos. Na Figura 14 é apresentada a análise de velocidade das partículas na zona de compressão do HPGR para os dois tipos de vedações com distância de 7,5 mm.

\section{Cheek plates}
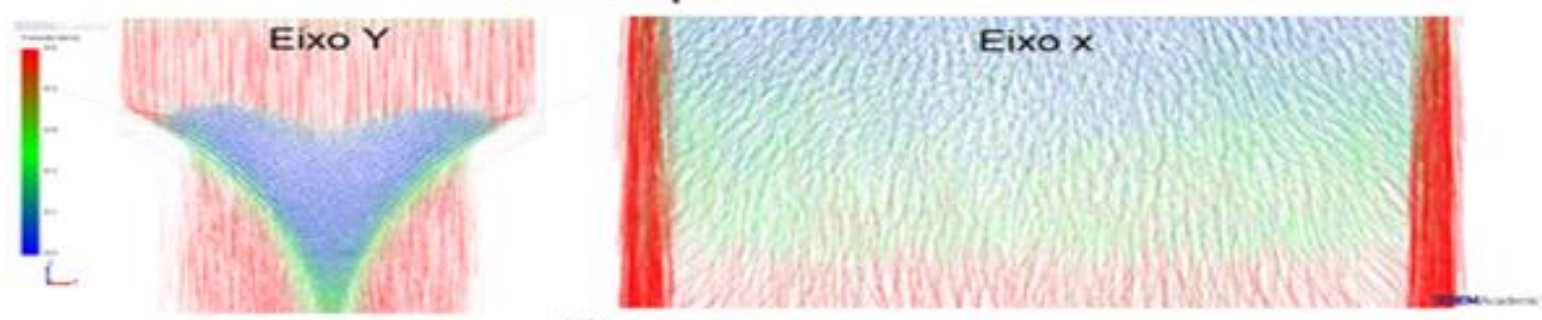

\section{Flanges}
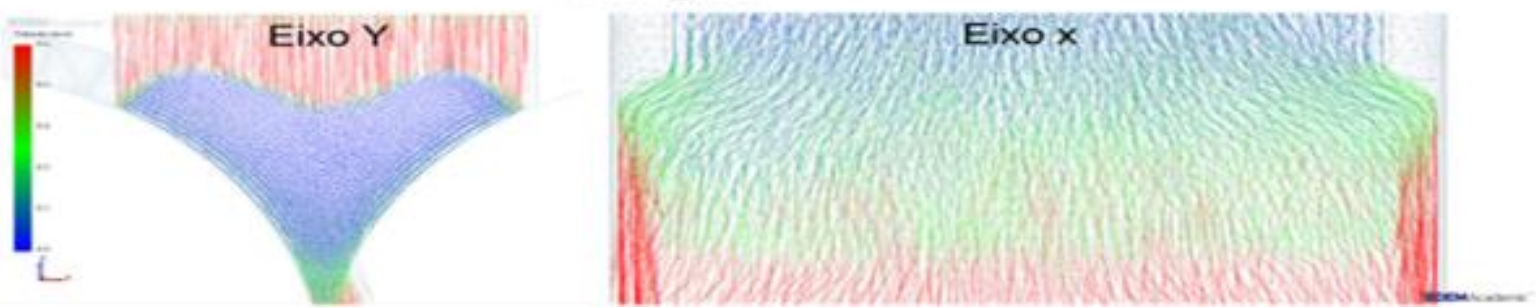
Figura 14. Visualização nos eixos $x$ (longitudinal) e y da velocidade das partículas para os dois tipos de vedações laterais com distância de $7,5 \mathrm{~mm}$.

Adicionalmente foi analisado o fluxo mássico de partículas através dos rolos. As Figuras 15 e 16 mostram a porcentagem de partículas que atravessam cada seção longitudinalmente no rolo, com vedações a distâncias de $7,5 \mathrm{~mm}$ e 2,0 $\mathrm{mm}$, respectivamente.

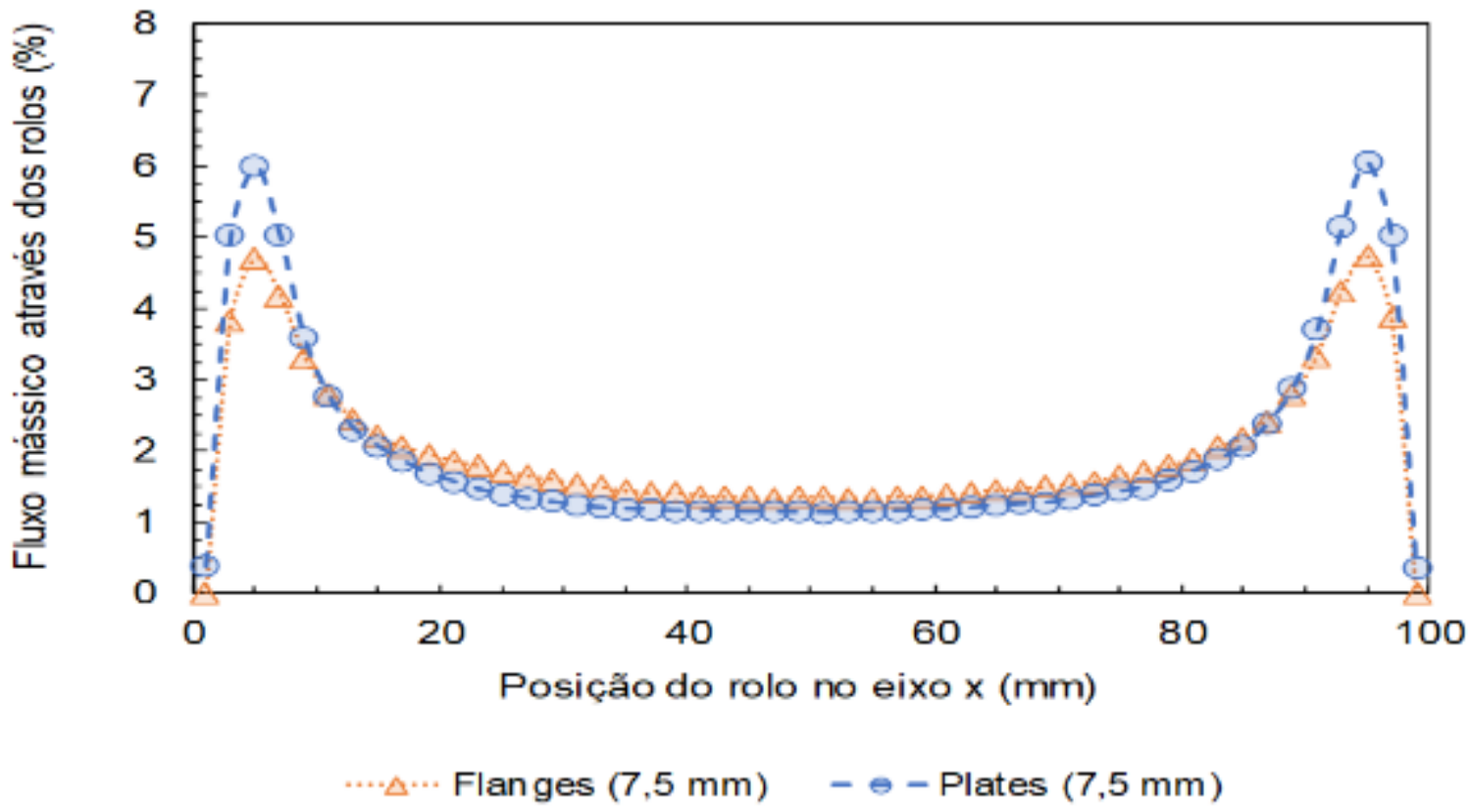

Figura 15. Fluxo mássico de partículas ao longo do eixo $x$ do rolo para vedações com distância de $7,5 \mathrm{~mm}$

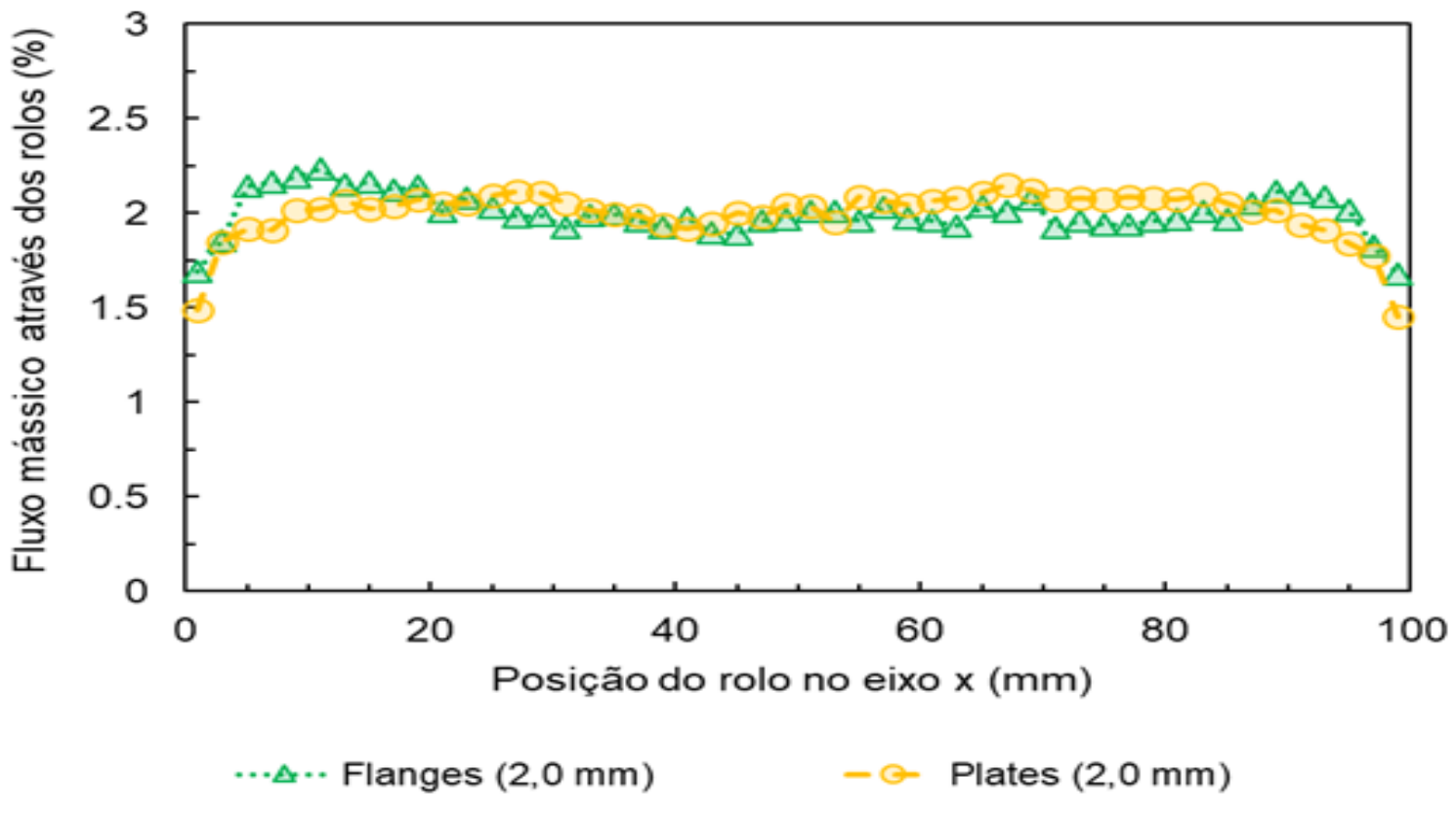

Figura 16. Fluxo mássico de partículas ao longo do eixo $x$ do rolo para vedações com distância de $2,0 \mathrm{~mm}$.

A amostragem em usina industrial permitiu quantificar o efeito de borda em relação à elevação de superfície específica, onde verifica-se um menor ganho nas bordas em relação ao centro do rolo. Pode-se verificar também que este perfil compromete a 
qualidade do produto final. Estes resultados então apresentados na Figura 17, na qual os pontos representam as posições de amostragem longitudinalmente abaixo do rolo (Figura 9).

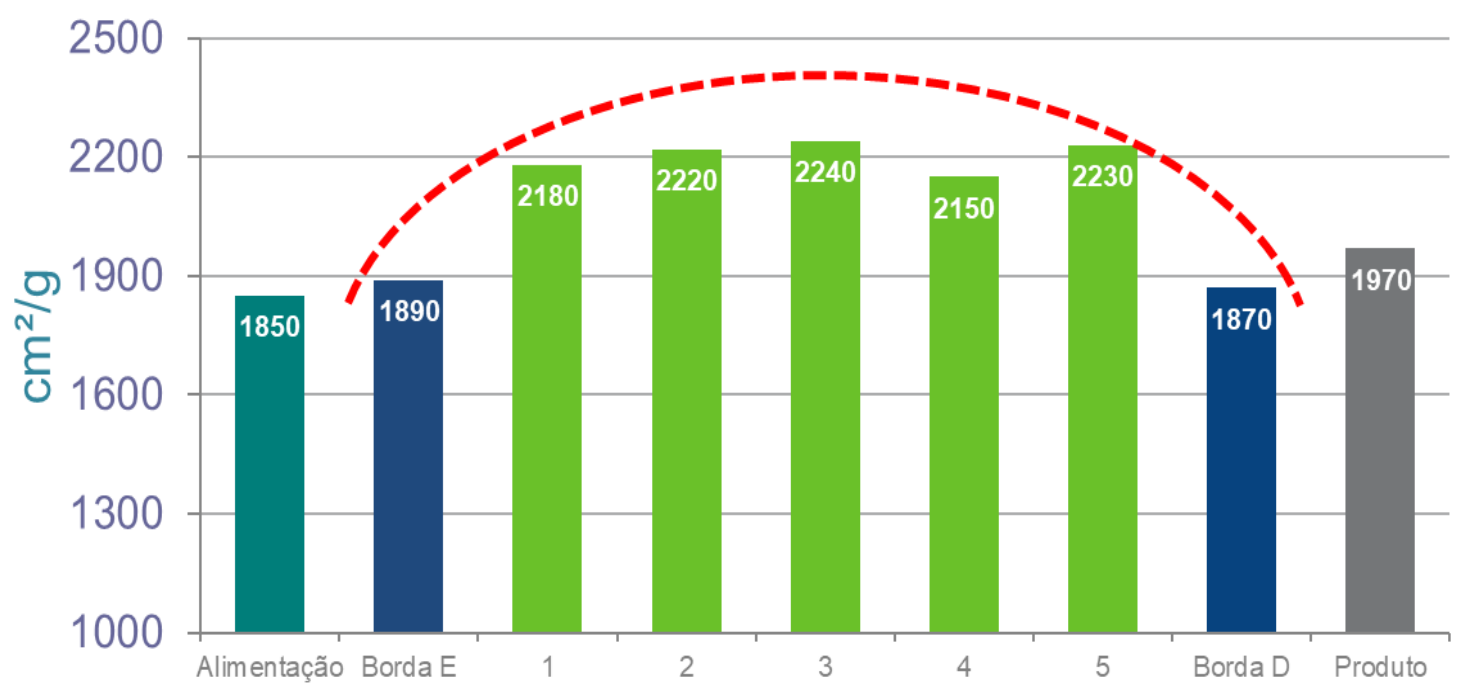

Figura 17. Efeito de borda em experimento industrial - Fonte: BUENO e VIANNA (2016).

\section{CONCLUSÃO}

$\checkmark$ A simulação de DEM com o software EDEM ${ }^{\circledR}$ permitiu reproduzir de forma bastante realista o fenômeno estudado;

$\checkmark$ Os resultados da simulação mostraram que o perfil característico do efeito de borda decorre do fato de não haver uma vedação perfeita entre os mecanismos de contenção das partículas com os rolos;

$\checkmark$ A vedação com Flanges se mostrou mais capaz de equalizar o perfil de pressões quando comparadas aos Cheek Plates;

$\checkmark$ Para um melhor desempenho da prensa de rolos, é necessária uma boa vedação nas laterais, de modo a deixar escapar o menor volume de material possível;

$\checkmark$ Com o experimento industrial foi possível quantificar o efeito de borda e sua contribuição para degradação do ganho do produto final influenciado pelo baixo ganho nas bordas, demonstrando-se a importância de garantir uma boa contenção lateral para o ótimo desempenho da prensa de rolos.

\section{REFERÊNCIAS}

ALVES V. K., Metodologia para simulação e escalonamento de prensas de rolos, Tese de Doutorado, UFMG, 2012.

BARRIOS G.K.P. Modelagem da prensa de rolos usando o método dos elementos discretos com acoplamento dinâmico e o modelo de substituição de partículas. Tese de Doutorado, UFRJ, 2015.

BARRIOS G.K.P., TAVARES L. M., A preliminary model of high pressure roll grinding using the discrete element method and multi-body dynamics coupling. International Journal of Mineral Processing 156 32-42, 2016. 
BUENO G., VIANNA, I., Guias laterais Nibrasco, Seminário interno Vale, Vitória - ES, 2016.

CARVALHO, R. M. DE. Desenvolvimento do Modelo Matemático generalizado da Cominuição. [s.I.] COPPE - UFRJ, 2009.

CARVALHO, R. M. DE. Simulation of Sag Mills Using a Mechanistic Model (J. Yianatos, R. Kuyvenhoven, Eds.)International Mineral Processing Congress. Anais...Santiago de Chile: 2014

CARVALHO R.M., BOECHAT F.O., Influência do tamanho de partículas para simulação pelo método dos elementos discretos em avaliação de um misturador horizontal, COPPE-UFRJ , 2016.

DA CUNHA, E. R.; DE CARVALHO, R. M.; TAVARES, L. M. Simulation of solids flow and energy transfer in a vertical shaft impact crusher using DEM. Minerals Engineering, v. 43-44, p. 85-90, 2013

DANIEL M.J., MORRELL S., HPGR model verification and scale-up, Minerals Engineering 17 1149-1161, 2004

DEM-SOLUTIONS. EDEM 2.7, 2016

METSO, HRC_Brochure, A evolução da tecnologia HPGR, 2013.

MISHRA, B. K.; RAJAMANI, R. K. The discrete element method for the simulation of ball mills. Applied Mathematical Modelling, v. 16, n. 11, p. 598-604, nov. 1992.

POLYSIUS AG, Desenho ํㅜ 20034211 NIBRAWAL, 2009

POLYSIUS AG, Laboratory high pressure grinding roll POLYCOM®, 2006

TAVARES L.M., Particle weakening in high-pressure roll grinding. Minerals Engineering, $18651-657,2005$.

TAVARES, L. M.; DE CARVALHO, R. M. Modeling breakage rates of coarse particles in ball mills. Minerals Engineering, v. 22, n. 7-8, p. 650-659, 2009.

TAVARES, L. M.; DE CARVALHO, R. M. Modeling ore degradation during handling using continuum damage mechanics. International Journal of Mineral Processing, v. 101, n. 1-4, p. 21-27, nov. 2011.

TORRES M., CASALI A., A novel approach for the modelling of high-pressure grinding rolls, Minerals Engineering, 22 1137-1146, 2009.

WATSON, S., BROOKS, M., KCGM evaluation of high pressure grinding roll technology. Fifth Mill Operators_Conference, Roxby Downs SA, pp. 69-83, 1994.

VAN DER MEER F.P., GRUENDKEN A., Flowsheet considerations for optimal use of high pressure grinding rolls. Minerals Engineering, 23 663-669, 2010 